\title{
Statistics based algorithm for exact quantitation of [biotechnology] seed traits without reference material
}

\begin{abstract}
An approach to the exact quantitative analysis of seed traits without using reference material is proposed basing on the quantum (discreet) nature of seed distribution is seed lots. The approach has been proven theoretically and supported experimentally with the analysis of the presence of seeds of soybean lines A2704-12, A5547-127 and GTS 40-3-2 in seed lots with the use of PCR in real-time mode tracing the transformation events. The approach may be applied not to PCR techniques only but to seed testing with whatever physical method provide the observed signal is obtained in exact values and the correlation between the signal and concentration of traced seeds is known. The approach can be of significant value in the issues of adventitious/technically unavoidable presence (AP), labeling, low level presence (LLP) and thus significantly simplify and decrease the cost of seeds breeding and trade.
\end{abstract}

Keywords: Biotechnology seeds, seed testing, quantitative analysis, hypergeometric distribution, statistical algorythm, real-time PCR, certified reference materials, selfreference
Volume 8 Issue 5 - 2019

\author{
Alexander Golikov, Pavel Frantsuzov, Maxim \\ Nikitin, Eugenia Strebulaeva, Oleg Tarakanov \\ GenBit LLC, Russian Federation
}

\begin{abstract}
Correspondence: Alexander Golikov, Ph.D., GenBit LLC, Varshavskoye highway, 28A, premise XIIIA, Moscow, II7I05 Russian Federation, +7 (926) 202-84-52, Email golikov@genbitgroup.com
\end{abstract}

Received:September 03, 2019 | Published: September 13, 2019

\section{Abbreviations}

GMO, genetically modified organism; CRM, certified reference material; DNA, desoxyribonucleic acid; PCR, polymerase chain reaction; LLP, low level presence; AP, adventitious presence; GM, genetically modified; FAM, 6-carboxyfluorescein; ROX, rhodamine $\mathrm{X}$; MGB, minor groove binder; BHQ1, BHQ2, black hole quenchers 1 and 2; CI, confidence interval

\section{Introduction}

The common and globally approved method of quantitative analysis of biotechnology traits/genetically modified organisms (GMO) is PCR in real time mode when the value of threshold cycle $\left(C_{t}\right)$ of tested sample is compared with $C_{t}$ values obtained through sequential dilution of the corresponding certified (standard) reference material (CRM) presented in the form of linear regression in semilogarithmic co-ordinates $\mathrm{C}_{t}$ vs $\log \left[\right.$ concentration, \%]. ${ }^{1}$ There are recent developments on using digital PCR with direct DNA copy counting, ${ }^{2}$ it also requires CRM though. However, certified reference materials may be quite expensive and not easily available, if available at all. Therefore, a method providing for exact quantitation of seed traits without using reference standards can be highly demanded.

ISTA (International Seed Testing Association) has developed a statistical tool (SeedCalc) ${ }^{3}$ using to assess seed purity/impurity, including level of GMO presence in conventional seed lots with qualitative testing plans using a Bayesian approach, so the results are statistical approximation. One of SeedCalc testing plans implies that a seed lot (sample) of 3000 seeds including unknown number of target seeds (seeds, that are subject to detection and differ in detected traits from the main bulk). The lot is then randomly distributed into 20 aliquots (subsamples) and the presence of target seeds is estimated basing on the number of positive aliquots (aliquots that gave positive result for the presence of target seeds).

However, real-time PCR provides exact figures for threshold cycle as the well-established function of the concentration of the tested object. Thus, we assume it is possible to make exact quantitative analysis once the exact number of target seeds in at least one aliquot or exact increment between a pair of aliquots is known. Seed lot is a discrete system, which means that the target seed content (in the seed count mode) cannot be fractional and the minimum possible content being exactly one seed. The minimum increment is also exactly one seed.

We therefore assume that within the range 1-150 target seed content per lot of 3000 seeds, randomly distributed in 20 aliquots, there will be an aliquot containing exactly one target seed or/and a pair of aliquots differing by exactly one target seed (with the probability not less than 0.95). The choice of 150 target seeds as the upper limit will be considered later in this paper, though it is more than satisfactory for the vast majority of issues related to seed purity, low level presence (LLP), adventitious presence (AP) and labeling.

\section{Theory}

For simplicity in mathematical considerations we will redefine terms seeds and aliquots so that seed=ball; target seed=red ball; non-target seed=white ball; aliquot=basket; positive aliquot (aliquot containing target seeds and showing detected trait/signal)=positive basket; negative aliquot (aliquot with zero content of target content not showing detected trait/signal $)=$ negative basket.

Thus, the starting condition is such that there are 3000 balls composed of $\mathrm{N}$ red balls and $(3000-\mathrm{N})$ white balls. The balls are randomly distributed into 20 baskets having 150 balls each.

Problem 1. Find probability $\mathrm{P}_{1}(\mathrm{~N})$ for $1 \leq \mathrm{N} \leq 150$ that the basket with the lowest non-zero number of red balls contains exactly one red ball.

Problem 2. Find probability $\mathrm{P}_{2}(\mathrm{~N})$ for $1 \leq \mathrm{N} \leq 150$, that two randomly selected baskets with a non-zero number of red balls contain $\mathrm{n}$ and $\mathrm{n}+1$ red balls, respectively.

To enumerate ways of distributing the balls, it is convenient to consider that 3000 balls are already distributed into 20 baskets, and 
randomly selected $\mathrm{N}$ balls are the red ones. In combinatorics such a selection is called combination: ${ }^{4}$

$$
\left(\begin{array}{c}
3000 \\
N
\end{array}\right)=\frac{3000 !}{N !(3000-N) !}
$$
Thus, total number of possible distributions $\operatorname{Total}(\mathrm{N})=\left(\begin{array}{c}3000 \\ \mathrm{~N}\end{array}\right)$
and this number grows quickly with $\mathrm{N}$.

Similarly, the number of possible distributions of $n$ red balls to the first basket is $\left(\begin{array}{c}150 \\ \mathrm{n}\end{array}\right)$. However, the number of possible distributions of $\mathrm{N}$ red balls when the first basket gets $n$ red balls is equal to the number of distributions of $n$ red balls to first basket multiplied by the number of distributions of $(\mathrm{N}-\mathrm{n})$ red balls to remaining baskets:

$$
\left(\begin{array}{c}
150 \\
\mathrm{n}
\end{array}\right)\left(\begin{array}{c}
3000-150 \\
\mathrm{~N}-\mathrm{n}
\end{array}\right)
$$

Using these formulas, we can find probability $P^{\prime}{ }_{N}(n)$ that the first basket gets exactly $n$ red balls:

$$
\mathrm{P}_{\mathrm{N}}^{\prime}(\mathrm{n})=\frac{\left(\begin{array}{c}
150 \\
\mathrm{n}
\end{array}\right)\left(\begin{array}{c}
3000-150 \\
\mathrm{~N}-\mathrm{n}
\end{array}\right)}{\left(\begin{array}{c}
3000 \\
\mathrm{~N}
\end{array}\right)}
$$

which is exactly hypergeometric distribution. ${ }^{5}$

Similarly, probability $\mathrm{P}^{\prime}\left(\mathrm{n}_{1}, \mathrm{n}_{2}, \ldots, \mathrm{n}_{\mathrm{k}}\right)$ that first $\mathrm{k}$ baskets get $\mathrm{n}_{1}, \mathrm{n}_{2}, \ldots, \mathrm{n}_{\mathrm{k}}$ red balls, respectively, can be calculated using the following formula:

$$
\mathrm{P}_{\mathrm{N}}^{\prime}\left(\mathrm{n}_{1}, \mathrm{n}_{2}, \ldots, \mathrm{n}_{\mathrm{k}}\right)=\frac{\left(\begin{array}{c}
150 \\
\mathrm{n}_{1}
\end{array}\right)\left(\begin{array}{c}
150 \\
\mathrm{n}_{2}
\end{array}\right) \ldots\left(\begin{array}{c}
150 \\
\mathrm{n}_{\mathrm{k}}
\end{array}\right)\left(\begin{array}{c}
3000-\mathrm{k}^{*} 150 \\
\mathrm{~N}-\mathrm{n}_{1}-\mathrm{n}_{2}-\ldots-\mathrm{n}_{\mathrm{k}}
\end{array}\right)}{\left(\begin{array}{c}
3000 \\
\mathrm{~N}
\end{array}\right)}
$$$$
\text { As a consequence, for } \mathrm{n}_{1}+\mathrm{n}_{2}+\ldots+\mathrm{n}_{20}=\mathrm{N}
$$$$
\mathrm{P}_{\mathrm{N}}^{\prime}\left(\mathrm{n}_{1}, \ldots, \mathrm{n}_{20}\right)=\frac{\left(\begin{array}{c}
150 \\
\mathrm{n}_{1}
\end{array}\right)\left(\begin{array}{c}
150 \\
\mathrm{n}_{2}
\end{array}\right) \ldots\left(\begin{array}{c}
150 \\
\mathrm{n}_{20}
\end{array}\right)}{\left(\begin{array}{c}
3000 \\
\mathrm{~N}
\end{array}\right)}
$$

gives us the formula for the probability of each individual distribution of 3000 balls ( $\mathrm{N}$ of which are red) into 20 baskets.

(Note that this problem is different from problem of randomly distributing $N$ red balls into 20 baskets, because the requirement of having exactly 150 balls in each basket affects resulting probability, so we cannot simply consider red balls alone and disregard white balls).

Using the previous formula, we get a simple brute-force method of calculating the probability of any event $E\left(n_{i}\right)$, which is symmetrical (does not depend upon the order of baskets), that is:

$$
E\left(\ldots, n_{i}, \ldots, n_{j}, \ldots\right)=E\left(\ldots, n_{j}, \ldots, n_{i}, \ldots\right) \text { for any } i, j:
$$

$\mathrm{P}(\mathrm{N} \mid \mathrm{E})=\sum\left\{\mathrm{P}_{\mathrm{N}}^{\prime}\left(\mathrm{n}_{1}, \ldots, \mathrm{n}_{20}\right): \mathrm{n}_{1}+\ldots+\mathrm{n}_{20}=\mathrm{N} ; \mathrm{E}\left(\mathrm{n}_{\mathrm{i}}\right)\right.$ holds true $\}$

The number of terms in this formula grows exponentially so it is impractical to use it for $\mathrm{N}$ over 20 , but we can notice that it is symmetrical with respect to permutations of $n_{1}, \ldots, n_{20}$. It means that we can only consider elements with $\mathrm{n}_{1} \leq \mathrm{n}_{2} \leq \ldots \leq \mathrm{n}_{20}$ and multiply each term by the number of ways this array of $n_{i}$ can be permutated.

In case $n_{i}$ numbers are all different the number of permutations will be 20 ! However, if there is a group of $g_{i}$ numbers that are the same, we need to divide the result by $\left(\mathrm{g}_{\mathrm{i}} !\right)$ because permutations of numbers in the same group yield the same sequence $\left\{n_{i}\right\}$. For example, in the sequence $\{0,0,0,1,1,3\} \mathrm{g}_{1}=3$ (three zeroes), $\mathrm{g}_{2}=2$ (two ones), $g_{3}=1$ (one three).

Finally, if we split $n_{1}, \ldots, n_{20}$ into groups of equal numbers, each consisting of $g_{1}, \ldots, g_{k}$ groups, then the final multiplier will be

$$
\frac{20 !}{\mathrm{g}_{1} ! \ldots \mathrm{g}_{\mathrm{k}} !}
$$

So, the final optimized brute force formula (master-formula) will be

$$
\begin{aligned}
& \mathrm{P}(\mathrm{N} \mid \mathrm{E})=\sum \frac{20 !}{\mathrm{g}_{1} ! \ldots \mathrm{g}_{\mathrm{k}} !} \mathrm{P}^{\prime}\left(\mathrm{n}_{1}, \ldots, \mathrm{n}_{20}\right): \\
& \mathrm{n}_{1}+\ldots+\mathrm{n}_{20}=\mathrm{N} ; \\
& \mathrm{n}_{1} \leq \mathrm{n}_{2} \leq \ldots \leq \mathrm{n}_{20} ; \\
& \mathrm{E}\left(\mathrm{n}_{\mathrm{i}}\right) \text { holds true; } \\
& \mathrm{n}_{\mathrm{i}} \text { form groups } \mathrm{g}_{1}, \ldots, \mathrm{g}_{\mathrm{k}} \text { of same numbers }
\end{aligned}
$$

Number of terms is much smaller in the optimized formula. Although exponential growth is still observed, it possible to calculate $\mathrm{P}(\mathrm{N} \mid \mathrm{E})$ for $\mathrm{N} \leq 150$ within reasonable time.

Computer calculations in exact rationales with arbitrary-precision arithmetic (https://en.wikipedia.org/wiki/Arbitrary-precision arithmetic) were employed, thus each $\mathrm{P}_{\mathrm{I}}(\mathrm{N})$ was found as exact rationale $\frac{\mathrm{P}}{\mathrm{Q}}, \mathrm{P}$ and $\mathrm{Q}$ being arbitrary-long integers, e.g.,

$$
P_{I}(60)=\frac{849357140474701955570725932674291143871481798931223756396220646710402270220898162849890399983083873059513964034014269000}{874008547796005305715880865842398785453190784485865613042898799660829699170076837903820651726749051888238144179924960953} \cong 0.9718
$$

Calculations were done in Microsoft Visual Studio, using C\# language, BigInteger (https://docs.microsoft.com/en-us/dotnet/ api/system.numerics.biginteger?view=netframework-4.8) and algorithms from BigRational library https://www.nuget.org/packages/ BigRationalLibrary/). The exact rationales were cast to normal floating-point numbers for the resulting data at the last stage only, thus, calculation precision was never lost

All the exact results obtained in this work were checked with Monte Carlo method. ${ }^{6}$ Ten thousand random permutations of an array containing 3000 elements - $(3000-\mathrm{N})$ zeroes and $\mathrm{N}$ ones-have been generated for each problem for each $\mathrm{N}$ using Fisher-Yates shuffle algorithm. ${ }^{7}$ Then first 150 elements of the array were assumed to be the first basket, next 150 elements being the second basket, etc. The simulated counts of the red balls $\mathrm{n}_{1}, \ldots, \mathrm{n}_{20}$ were obtained by counting number of ones in each of these baskets. Then the condition was checked on $\mathrm{n}_{1}, \ldots, \mathrm{n}_{20}$, and the resulting probability was calculated as a number of positive outcomes divided by number of simulations (the latter being equal to 10000). 
The difference between the exact results obtained and Monte Carlo simulations varied in each case but never exceeded $0.4 \%$.

Results obtained with the use of the above master-formula are given in Figure 1.

It is clearly seen from Figure 1 that the assumption of always having a basket (aliquot) with exactly one red ball (target seed), or/and two baskets with non-zero content differing by exactly one red ball (target seed) is correct for any $1 \leq \mathrm{N} \leq 150$. The probability never goes under 0.99 except for $\mathrm{N}=2$ and never goes under 0.999 for $6 \leq \mathrm{N} \leq 150$. Indeed, two red balls can be distributed in two ways only, i.e., two balls in one basket (probability $\sim 0.0497$ ) and two baskets with one ball in each (probability 0,9503). Still it is above 0.95 .

Figure 2 gives the range of $\mathrm{N}$ red balls (target seeds) occurrence as function of the nubmer of positive baskets (aliquots) observed.

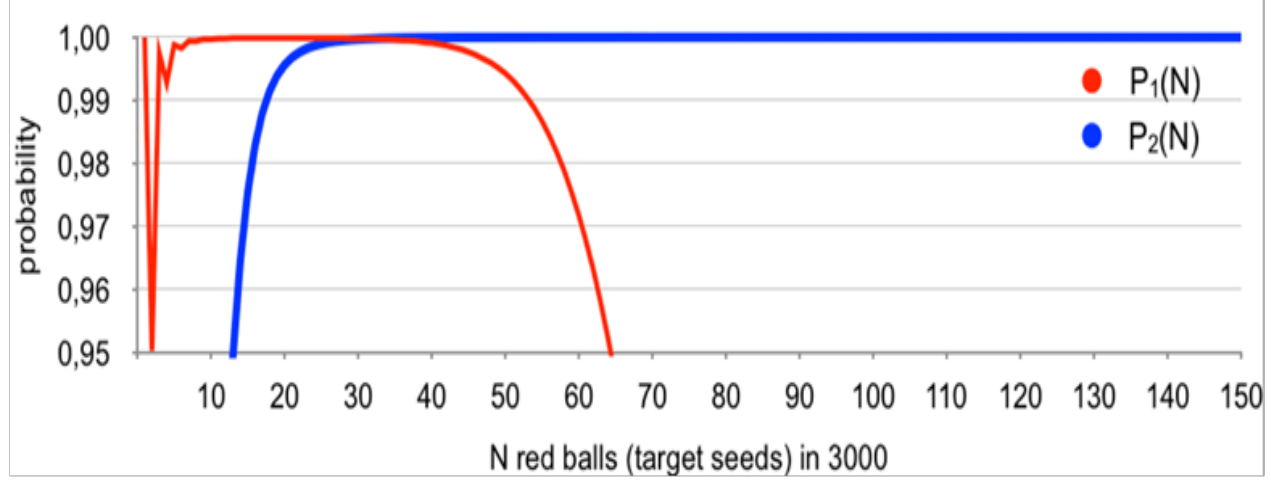

Figure I Probability of occurrence of a basket (aliquot) with exactly one red ball (target seed) $\mathrm{P}_{\mid}(\mathrm{N})$; probability of having an increment of exactly one red ball (target seed) between two randomly selected baskets (aliquots) $P_{2}(N)$.

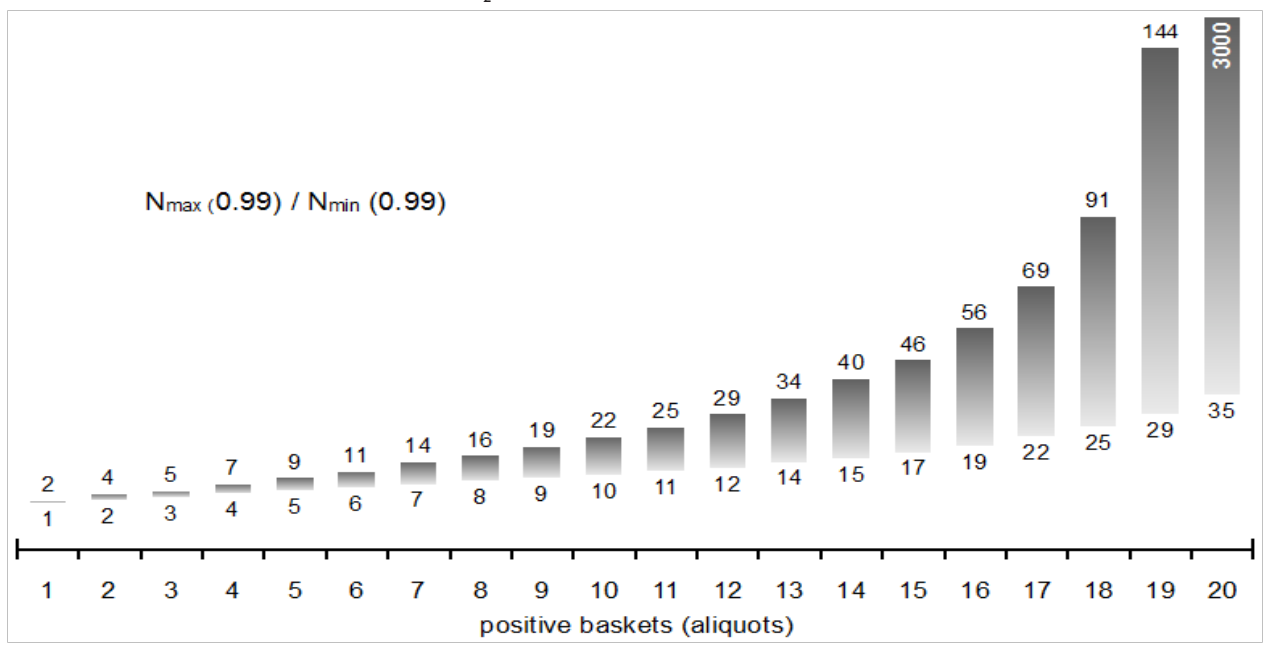

Figure 2 Range of occurrence of red balls (target seeds) in positive baskets (aliquots).

$\mathrm{N}_{\min }(0.99)$ and $\mathrm{N}_{\max }(0.99)$ mean, that, if, for example we have 15 positive baskets, we can expect 17 to 46 red balls. Probability of finding $\mathrm{N}=16\left(\mathrm{~N}_{\min }(0.99)-1\right)$ or $\mathrm{N}=47\left(\mathrm{~N}_{\max }(0.99)+1\right)$ in 15 baskets is lower than 0.01 . It can be clearly seen from Figure 2 that there is huge uncertainty in binding a particular number of positive baskets (aliquots) to the occurrence of a particular N, e.g., 30 red balls (target seeds) can be observed within the range 13-19 positive baskets (aliquots). It is obvious therefore that effective algorithm is needed to identify which basket (aliquot) or two baskets (aliquots) should be selected depending on the number of positive baskets (aliquots) observed, so the "exactly one" criterion is applied correctly.

In a series of computer simulations and experiments we came to the following:

\section{Algorithm}

Let $\mathrm{K}$ be the number of positive aliquots and two positive aliquots differ when the difference in their signals $\left(C_{t}\right.$ in the case of real-time
PCR) goes beyond standard deviation values, i.e.,

$$
C_{t}(i) \neq C_{t}(j) \text { when }\left|C_{t(i)}-C_{t(j)}\right|>\sigma_{i}, \sigma_{j} .
$$

Sort (arrange) positive baskets (aliquots) so, that they are indexed $b_{1}, b_{2} \ldots, b_{k}$, where $b_{1}$ has the minimum number of the target seeds and $b_{k}-$ the maximum (in case of real-time PCR it means sorting from highest $\mathrm{C}_{\mathrm{t}}$ value to lowest).

There are several special issues to be addressed at the starting point:

1. $\mathrm{K}=1$, then $\mathrm{N}$ (total number of target seeds) is most likely 1 or 2 (with the probability below 0.05 for $\mathrm{N}=2$ ). $\mathrm{N}>2$ for one positive aliquot is most unlikely-the probability of having 3 and 4 target seeds in is less than 0.0025 and 0.000125 , respectively.

2. $2 \leq \mathrm{K} \leq 12$ and all aliquots show the same signal $\left(\mathrm{C}_{\mathrm{t}}\right)$. Then target seeds are distributed equally by one target seed per aliquot $(1,1$; $1,1,1 ; 1,1,1,1$, etc.). Probability of any other equal distribution 
is extremely low. Thus, for $\mathrm{K}=2$ probability of 2,2 and 3,3 combinations is lower than 0.007 and 0.000125 , respectively. With higher $\mathrm{N}$ and $\mathrm{K}$, the probability of equal distribution becomes negligibly low quickly, e.g., the probability of 2,2,2,2,2 distribution for $\mathrm{K}=5$ is less than 0.0002 .

$\mathrm{K}=20$ and all aliquots show the same signal $\left(C_{t}\right)$. Then $\mathrm{N}>150$.

We now shall consider the core case only, when we have a series of positive baskets (aliquots) with differing signal $\left(C_{t}\right.$ for real-time $\left.P C R\right)$ values.

There are four problems to be solved:

Find probability $P_{I}(N)$ for $1 \leq N \leq 150$ that basket $\mathrm{b}_{1}$ contains exactly one red ball.

Find probability $P_{I I}(N)$ for $1 \leq N \leq 150$, that basket $\mathrm{b}_{1}$ and the next basket with the second lowest red balls content contain exactly one and two red balls, respectively.

Find probability $P_{I I I}(N)$ for $1 \leq N \leq 150$, that basket $\mathrm{b}_{1}$ and the next basket the second lowest red balls content contain $\mathrm{n}$ and $(\mathrm{n}+1)$ red balls, respectively

Define the median $\mathrm{M}$ as $\mathrm{K} / 2$. Then the pre-median basket will have index $(K / 2)$ if $K$ is even or $(K / 2-0.5)$ when $K$ is odd. Find probability $P_{I V}(N)$ for $1 \leq N \leq 150$, that the pre-median basket and the post- median basket (closest to $\mathrm{M}$ on the higher side and differing from the pre-median basket in red balls content) have $\mathrm{n}$ and $(\mathrm{n}+1)$ red balls, respectively $(\mathrm{n} \neq 0)$.

This brings us to the main problem: 3000 balls containing $\mathrm{N}$ red balls and $(3000-\mathrm{N})$ white balls are randomly distributed into 20 baskets having 150 balls each. The exact value of $\mathrm{N}$ (number of red balls in the 3000 balls lot) is unknown, but we consider it within the range $1 \leq N \leq 150$.

Consider the following algorithm:

\begin{tabular}{ll}
\hline $\begin{array}{l}\text { Number of baskets } \\
\text { with no red balls }\end{array}$ & Then assume that... \\
\hline a) $4-20$ & $\begin{array}{l}\text { the basket with the minimal number of red balls } \\
\text { contains exactly one red ball }\end{array}$ \\
b) 2 or 3 & $\begin{array}{l}\text { the two baskets with minimal number of red } \\
\text { balls differ by exactly one red ball }\end{array}$ \\
c) 0 or I & $\begin{array}{l}\text { two baskets positioned right below and above } \\
\text { the median (the pre-median and post-median } \\
\text { ones) differ by exactly one red ball }\end{array}$ \\
\hline
\end{tabular}

Find probability $\mathrm{P}(\mathrm{N})$ that the proposed algorithm is correct. Prove $P(N) \geq 0,95$ for any $1 \leq N \leq 150$.

Results obtained with the use of the master-formula described earlier are given in Figures 3,4.

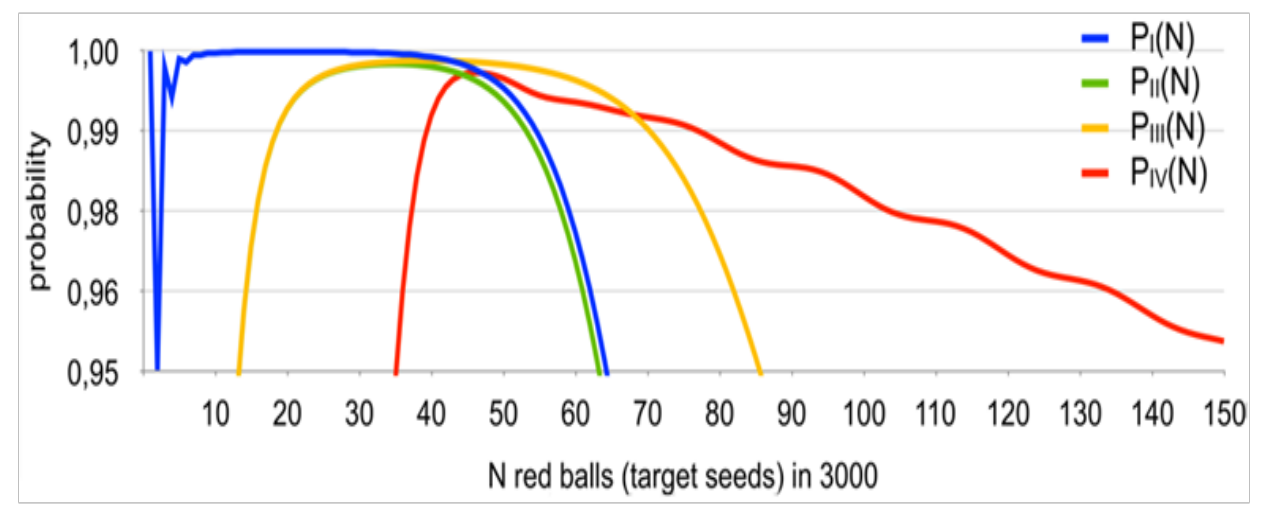

Figure 3 Probability of basket (aliquot) \#I containing exactly one red ball (target seed) - $\mathrm{P}_{1}(\mathrm{~N})$; probability of basket (aliquot) \#I and the next basket (aliquot) with the second lowest red balls (target seeds) content containing exactly I and 2 red balls (target seeds), respectively - $P_{\| \prime}(N)$; probability of basket (aliquot) $\mathrm{\# I}$ and the next basket (aliquot) with the second lowest red balls (target seeds) content containing $n$ and $(n+I)$ red balls (target seeds), respectively - $P_{I I I}(N)$; probability of the pre-median basket (aliquot) and the post-median basket (aliquot) have $n$ and $(n+I)$ red balls, respectively - $P_{I V}(N)$.

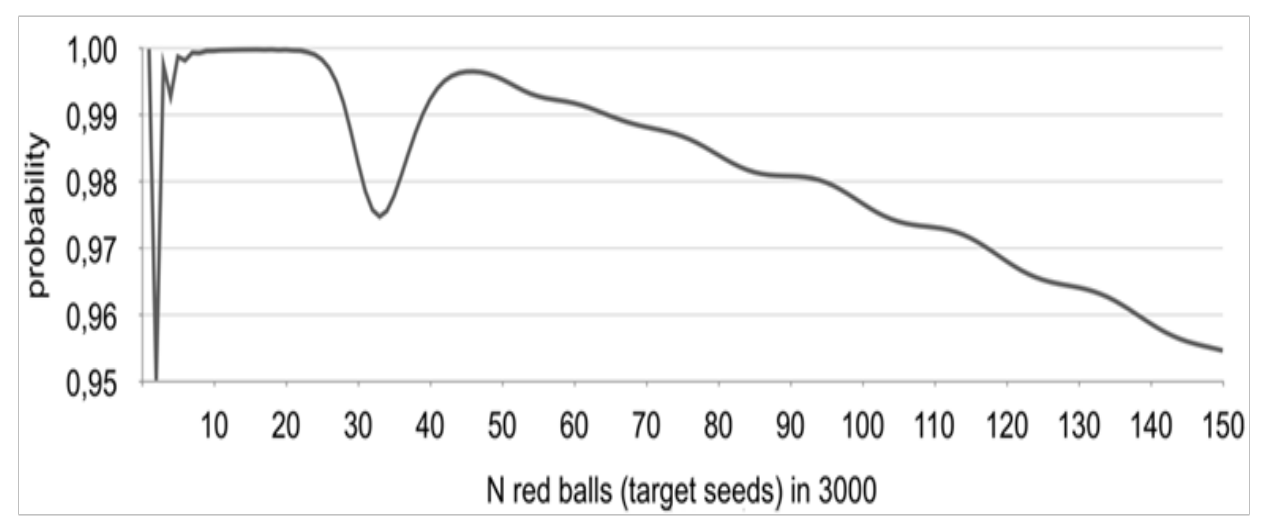

Figure 4 Probability of the proposed algorithm providing correct results - $P(N)$.

Note the dip in the probability between $N=30$ to 40 , relates to the fact that the probability of zero or one empty baskets is already non-negligible (2\% for $N=30)$, but $P_{I V}(N)$ is still small, violating option c) of the definition of $P(N)$ in almost $3 \%$ of all cases.. 
The areas of high probability intersect for the measurements:

$$
\begin{aligned}
& P_{I}(N) \geq 0,95 \text { for } 1 \leq N \leq 65 ; \\
& P_{I I}(N) \geq 0,95 \text { for } 14 \leq N \leq 64 ; \\
& P_{I I I}(N) \geq 0,95 \text { for } 14 \leq N \leq 85 ; \\
& P_{I V}(N) \geq 0,95 \text { for } 35 \leq N \leq 150
\end{aligned}
$$

are self-explanatory showing the range of possible presence of target seeds as function of the number of positive aliquots observed and give the possibility to cross-check experimental results obtained with different criteria of the algorithm above because it is obviously seen that more than one of "exactly one" criteria can be applied to the wide range of positive aliquots. Thus, both a) and b) criteria apply in case 2-16 positive aliquots, both b) and c) applies in case of 14-18 positive aliquots. And there is the special case that two aliquots with the lowest and second lowest target seed content have exactly one and two target seeds, respectively (applies to the range of 8-16 positive aliquots). This case will be discussed under "Experimental proof" below.

It clearly seen that $P(N)>0,95$ for $1 \leq N \leq 150$. The graph is not even because different trends of $P_{I}(N), P_{I I}(N)$ and $P_{I I I}(N)$ interact along with the decreasing probability of occurrence of baskets (aliquots) with no red balls (target seeds) while $\mathrm{N}$ grows.

Results given in Figure 4 justify the choice of using $\mathrm{N}=150$ as the upper limit in the approach since reliability of the algorithm has a trend to go below 0,95 . Nevertheless, the range 1-150 target seeds in 3000 seeds $(\sim 0.03 \%-5 \%)$ lot is more than enough for the vast majority of issues related to low limit presence, adventitious presence, labeling and seed purity.

Once it is proven that the presence of target seeds is exactly known for one known aliquot (or the difference in target seed content for a known pair of aliquots) we come to the equation of linear regression used in quantitative analysis. In the case of testing an aliquot with 150 seeds it is

$$
C_{t}=A \cdot \log [(n / 150) \cdot 100, \%]+B
$$

where $\mathrm{n}$ is the number of target seeds in the tested aliquot, which, after simple algebraic transformations gives

and

$$
\begin{aligned}
& \mathrm{n}_{\mathrm{i}}=\frac{\mathrm{n}_{1}}{\left(\frac{\mathrm{C}_{\mathrm{t}}(1)-\mathrm{C}_{\mathrm{t}}(\mathrm{i})}{\mathrm{A}}\right)} \\
& \mathrm{n}_{1}=\frac{1}{10^{\left(\frac{\mathrm{C}_{\mathrm{t}}(1)-\mathrm{C}_{\mathrm{t}}\left(1^{+}\right)}{\mathrm{A}}\right)}-1}
\end{aligned}
$$

In case of algorithm criterion a) when there are $2-16$ positive aliquots, $n_{1}=1$ and $C_{t}(1)$ refer to positive aliquot $\# 1$ (sorted and $n_{1}=1$; in case of criteria b) and c) when two aliquots differing by one target seed are selected, $\mathrm{n}_{1}$ and $\mathrm{C}_{\mathrm{t}}(1)$ refer to the first aliquot of the two-it can be aliquot $\# 1$ and the pre-median aliquot. $C_{t}\left(1^{+}\right)$ refers to the second aliquot of the chosen two, i.e., the one having one target seed more. Once calculated, $n_{i}$ shall be rounded to the nearest integer value because the number of seeds cannot be fractional.

The sum of target seeds in all positive aliquots gives the content of target seeds $(\mathrm{N})$ in the 3000 seeds lot

$$
\mathrm{N}=\sum_{\mathrm{i}=1}^{\mathrm{K}} \mathrm{n}_{\mathrm{i}}
$$

Standard protocol of quantitative analysis with the use of real-time PCR requires that constants A (slope) and B are derived through a series of dilution of certified (standard) reference material. However, the slope value (A) depends solely on the amplified DNA sequence (amplicon), oligonucleotides (primers and probes) used for testsystems, and PCR protocol. In other words, the slope is the same for the CRM and the tested sample. In fact, quantitative analysis using CRM is based upon this principle.

The slope value is then derived through a series of sequential dilution of the most populated aliquot (self-reference calibration). Using self-calibration is justified because we deal with the systems where all target seeds are well unified discreet pieces. Direct calculations checked with Monte Carlo method show that the ratio between target seeds content in the most populated aliquot $\left(\mathrm{n}_{\max }\right)$ and that in the least populated aliquot $\left(\mathrm{n}_{\min }\right)$ varies within the range $3 \leq \mathrm{N} \leq 150$ however, in most cases it is hardly expected to exceed 10 up to $\mathrm{N}=150$ ( $\sim 5 \%$ probability at the high end). Thus, the slope derived from dilution series in the range $\mathrm{n}_{\max }-0.1 \mathrm{n}_{\max }$ (ten-fold dilution) covers the full range of target seed presence in all positive aliquots, and $0.1 \mathrm{n}_{\max }$ is always above real-time PCR detection limit (0.1\%).

\section{Materials and methods}

\section{Seed material}

Beans of genetically modified soybean (GM-soybean) lines A270412 (event code ACS-GMØØ5-3) and A5547-127 (event code ACSGMØØ6-4) were provided by the FBUN "Federal Research Centre for nutrition and biotechnology" (Moscow, Russian Federation) within the framework for the development of the national technical guidelines "Detection and identification of GMO of plant origin with real-time PCR in matrix format" (MUK 4.2.3390-16) [https://www. rospotrebnadzor.ru/documents/details.php?ELEMENT_ID=8637].

Beans of GM-soybean line GTS 40-3-2 (event code MONØ4Ø32-6) (commodity soya beans imported from Paraguay), and non-GM soybean were provided by the "Group of Companies SODRUGESTVO” (Kaliningrad, Russian Federation).

All beans used in the experiment were calibrated to have the same weight $(\mathrm{SD}=1,5 \%)$.

\section{DNA extraction}

Blender 8010S (Waring Commercial, USA) was used to grind the beans into fine flour. $50 \mathrm{mg}$ of soybean flour taken with OHAUS AP210 balance (Ohaus, Switzerland) was used for a single extraction of genomic DNA with DNA AmpliSens extraction kit DNAsorb-C-M (FBUN "Central Research Institute of epidemiology of Rospotrebnadzor", Moscow, Russian Federation) in accordance with the producer's manual. DNA concentration was measured at $\lambda=260$ $\mathrm{nm}$ with SmartSpec Plus spectrophotometer (BioRad, USA). Purity of extracted DNA was checked by A260/A280 ratio. Extracted DNA specimens were stored at $-20^{\circ} \mathrm{C}$. 


\section{Oligonucleotides}

Primers and probes (with 5'-fluorescent FAM or ROX dyes) were produced by "Biotech-Industry" LLC (Moscow, Russian Federation). The probe with MGB modification was produced by Thermo Fisher Scientific (USA). All oligonucleotides used were in full compliance with JRC EC protocols, as follows:

\section{A2704-12}

fwd: GCAAAAAAGCGGTTAGCTCCT; rev: ATTCAGGCTGCGCAACTGTT;

probe: ROX-GGAAGGGCGATCGGAGGACCG-BHQ2

A5547-127

fwd: CTATTTGGTGGCATTTTTCC; rev: TGCGGCCAACTTACTTC

probe: FAM-ACAACGATGACGGTATGACATTGCGG-BHQ1

GTS 40-3-2 ${ }^{10}$

fwd: TTCATTCAAAATAAGATCATACATACAGGTT; rev: GGCATTTGTAGGAGCCACCTT; probe: FAM-CCTTTTCCATTTGGG-MGBNFQ

\section{$P C R$ in real-time mode}

PCR in real-time mode was carried out using the DTLite detecting thermocycler ("DNA-Technology" LLC, Moscow, Russian Federation). $25 \mu 1$ of reaction mixture included $2.5 \mu 1$ of $10 \mathrm{x}$ PCR buffer, $1,2 \mu 1 \mathrm{Mg}^{2+}(50 \mathrm{mM}), 0,6 \mu 1$ of dNTP $(10 \mathrm{mM})$ mixture, $2 \mu 1$ of each of the primers $(10 \mu \mathrm{M}), 2 \mu \mathrm{l}$ of the probe $(5 \mu \mathrm{M}), 0,5 \mu 1$ of TaqDNA-polymerase (5 U/ $\mu 1$, "SibEnzym" LLC, Novosibirsk, Russian Federation), $9,2 \mu 1$ of deionized water, and $5 \mu 1$ of the specimen DNA. Pipetting was done with Sartorius Picus ${ }^{\circledR}$ electronic pipet, 0.2-10 $\mu \mathrm{l}$ ("Sartorius GMBH", Germany).

PCR was carried out in the following mode: $50{ }^{\circ} \mathrm{C}-2 \mathrm{~min}$, $95{ }^{\circ} \mathrm{C}-10 \mathrm{~min}$, and 50 cycles of denaturation $-15 \mathrm{sec}$ at $95^{\circ} \mathrm{C}$ and annealing+elongation- $60 \mathrm{sec}$ at $60^{\circ} \mathrm{C}$. Data analysis and calculation of threshold cycles was done automatically with the program software RealTime PCR v7.9 ("DNA-Technology" LLC, Moscow, Russian Federation).

Each test was carried out in five repeats and was accompanied with positive and negative control reactions, when corresponding plasmid and deionized water, respectively, was added instead of the specimen DNA.

\section{Experiment setup}

Two 3000 seed lots have been formed for the experiment

a) 14 beans of soybean line A27014-12+16 beans of soybean line A5547-127+2970 beans of non-GM soybean;

b) 150 beans of soybean line GTS 40-3-2+2850 of non-GM soybean.

\section{Emulation of target seeds distribution}

Emulation was performed using plastic balls $3 \mathrm{~mm}$ in diameter differing in color (black, blue and red). 3000 balls-(16 red +14 blue +2970 black) emulating the mixture of A2704-12 and A5547-127, and (150 red+2850 black) emulating GTS 40-3-2. Mixture of 3000 balls was put into an opaque bag, thoroughly mixed, then random ball was taken out "blindly" by hand (like when playing lotto) and put into one of 20 numbered (1-20) glasses. Then the balls left in the bag were mixed again. The process was repeated until all 3000 balls were distributed into 20 aliquots, containing 150 balls each.

\section{Forming the aliquots}

Since all the seed material was commodity soybean, seeds were selected to have the same weight $( \pm 5 \%)$. Each seed was crushed into fine flour individually with pestle and mortar, genomic DNA was extracted from $50 \mathrm{mg}$ of the flour and tested for the presence of the target transformation event. The flour from selected seed was combined in one batch and thoroughly mixed. Then $100 \mathrm{mg}$ of the flour was defined as 1 seed. The real soybean aliquots were composed by reproducing the emulation above. Each $\mathrm{i}^{\text {th }}$ aliquot composition exactly repeated that in the emulated $i^{\text {th }}$ aliquot, where real seeds of A2704-12, A5547-127, GTS 40-3-2 soybean lines, and non-GM soybeans were used instead of red, blue and black balls. Thus, for example, in case of the aliquot with 3 seeds of line GTS 40-3-2 it contained $300 \mathrm{mg}$ of the flour from GTS 40-3-2 batch+14700 mg of the flour from nonGM soybean. Weighing was done with $1 \%$ accuracy to emulate live situation.

Each aliquot containing $15 \mathrm{~g}$ of fine soya flour was thoroughly mixed. Since the aliquot weight was $\sim 15 \mathrm{~g}$ and the sample for DNA extraction was $50 \mathrm{mg}$, there was a need to provide the best representation of recombinant DNA in the specimen, especially for the aliquots with low target seeds presence. We have therefore prepared the most possible diluted sample consisting of $100 \mathrm{mg}$ of GTS 40-3-2 flour and 14900 non-GM flour, mixed thoroughly and performed 30 extractions, thus having total DNA of the aliquot divided into equal parts. $\mathrm{C}_{\mathrm{t}}$ and standard deviation for the 30 specimens were obtained. Then we combined the single specimens into twos, threes, fours, fives, etc. (not less than 12 random combinations in each case) and found that $\mathrm{C}_{\mathrm{t}}$ and standard deviation values stopped changing with the number of combined single specimens over four. Thus, we used seven single extraction procedures for all of aliquots, combining the resulting single extracts of genomic DNA to provide best confidence in its correct presence in the specimen used for real-time PCR.

\section{Slope (A) estimation (self-reference calibration)}

Calibration line $\mathrm{C}_{\mathrm{t}} \mathrm{vs} \log [\mathrm{C}, \%]$ providing the slope (A) value was built using DNA extracted from the aliquot with the highest target seeds content, i.e., the one showing the lowest $\mathrm{C}_{t}$ value. Concentration of GM soybean in this aliquot was taken for $100 \%$. DNA of the specimen in this aliquot was then diluted with DNA of non-GM soybean to obtain points with $56.2 \%, 31.6 \%, 17,8 \%$ and $10 \%$ relative concentration of the initial concentration (just to have even distribution of points along $\log [\mathrm{C}]$ axis). Obtained DNA solutions were used as standards for building the calibration line $\mathrm{C}_{\mathrm{t}}$ vs $\log [\mathrm{C}, \%]$. $\mathrm{C}_{\mathrm{t}}$ was derived in five repeats for each calibration point.

\section{Results and discussion}

Raw data on $\mathrm{Ct}$, standard deviation and self-reference calibration, calculated and actual values are given in Table $1 \mathrm{~S}$.

\section{A2704-12}

Results for real-time PCR performance on A2704-12 are given in Figure 5 (in blue) and Table 1S. 
Correspondence between the actual and sorted order of the aliquots can be found in Table 1S.

It is clearly seen from Figure 5 that nine aliquots showed positive for A2704-12. According to data from Figure 2 this means that total number $(\mathrm{N})$ of A2704-12 beans in the 3000 lot is within 9-19 range and algorithm a) shall be applied-the aliquot with the least target seed content has exactly one target seed. It can be seen from Figure 5 that there are five aliquots (\#\# 1 to 5), that show maximum $C_{t}$ value, and the average $C_{t}$ for an aliquot with 1 target seed is 27,75 . The slope (A) value derived from the series of dilution of aliquot \#9 (earliest $\mathrm{C}_{\mathrm{t}}$ and highest A2704-12 content, respectively) is $-3.31 \pm 0,19$. The self-reference calibration line was true linear $\left(\chi^{2}=0,0651\right.$ and $\mathrm{R}^{2}=$ 0.9906).

Applying these figures to the formula for $\mathrm{n}$ calculation, we have the following:

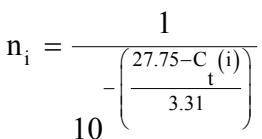

$n_{i}$ is then rounded to the nearest integer value, so we get the distribution (in the order of sorted aliquots) 1,1,1,1,1,2,2,2,3, which gives $\mathrm{N}=14$. And this is the exact match with the actual distribution in the seed lot, both for each aliquot and for the total presence of A2704-12.

Since A has standard deviation $\sigma_{A}=0,19$ we can calculate $n_{\min }\left(-A=-3,31-\sigma_{A}\right)$ and $n_{\max }\left(A=-3,31+\sigma_{A}\right)$ for each aliquot (in this particular case $\mathrm{n}$ values shall not be rounded). The normalized absolute deviation $\Delta_{\mathrm{i}}$ for each $n_{\mathrm{i}}$ will be

$$
\Delta_{\mathrm{i}}=\frac{\left|\mathrm{n}_{\text {max }}-\mathrm{n}_{\text {min }}\right|}{2 \mathrm{n}_{\mathrm{i}}} \text { and } \leq \sigma_{\mathrm{i}}=\sqrt{\frac{\sum \Delta_{\mathrm{i}}^{2}}{\mathrm{~K}-1}}
$$

where $\sigma_{n}$ is the normalized standard deviation and $\mathrm{K}$ is the number of positive aliquots.

Standard deviation for the total number of target seed in the aliquot is then $\sigma_{N}=\sigma_{n} K=0.33$ and

$$
N=14 \pm 0.33 ; N_{\text {actual }}=14
$$

SeedCalc gives $5 \leq N \leq 23$, Confidence Interval $(\mathrm{CI})=95 \%$ with the optimum at $\mathrm{N}=12$.

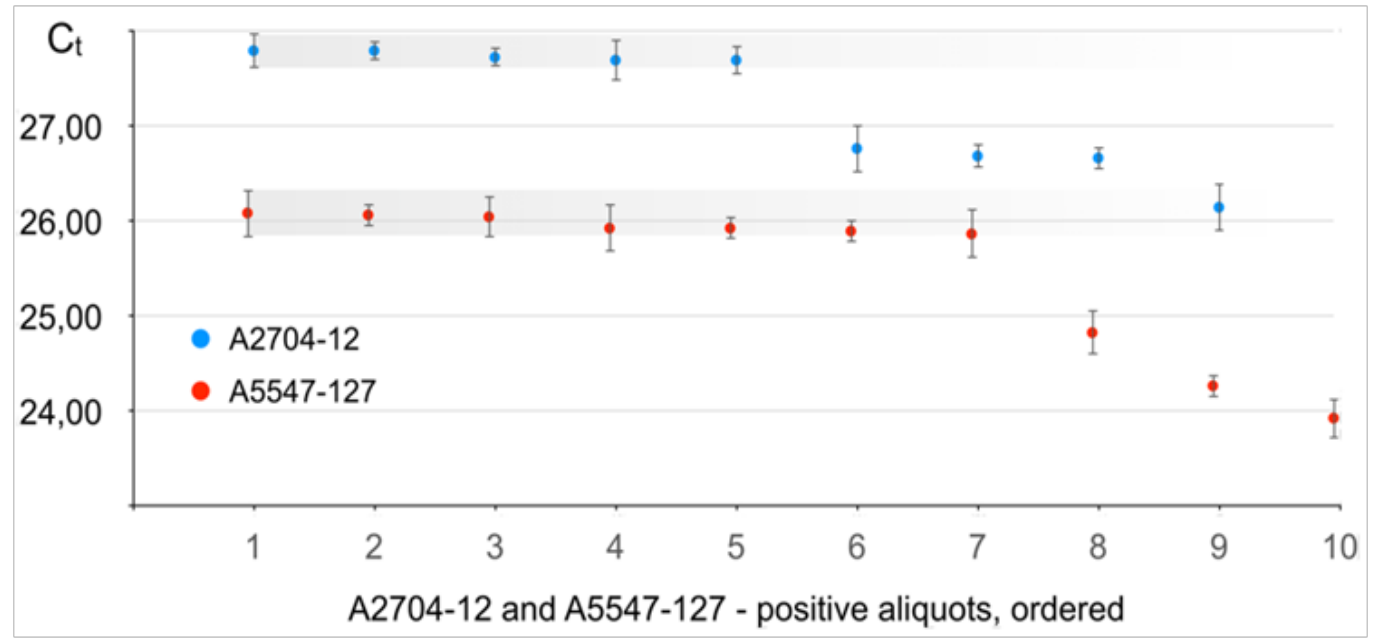

Figure 5 Real-time PCR performance on soybean lines A2704- 12 and A5547-127 in the seed lot of 3000 seeds distributed in 20 aliquots. Positive aliquots arranged in the order from the lowest target seed content (latest $C_{t}$ ) to the highest (earliest $\left.C_{t}\right)$. Error bars give standard deviation values $(\sigma)$ resulting from multiple repeats of testing of each aliquot (Table IS).

\section{A5547-127}

Results for real-time PCR performance on A5547-127 are given in Figure 5 (in red) and Table 1S.

Correspondence between the actual and sorted order of the aliquots can be found in Table $1 \mathrm{~S}$.

As it follows from Figure 5, ten aliquots showed positive for A5547-127. According to data from Figure 2 this means that total number $(\mathrm{N})$ of A5547-127 beans in the 3000 lot is within 10-22 range and algorithm a) shall be applied-the aliquot with the least target seed content has exactly one target seed. It can be seen from Figure 5 that there are seven aliquots (\#\# 1 to 7), that show maximum $C_{t}$ value, and the average $\mathrm{C}_{\mathrm{t}}$ for an aliquot with 1 target seed is 26,09 . The slope (A) value derived from the series of dilution of aliquot $\# 10$ (earliest $C_{t}$ and highest A5547-127 content, respectively) is $-3.50 \pm 0,16$. The selfreference calibration revealed true linear regression $\left(\chi_{2}=0,0409\right.$ and $R^{2}=0.9947$ ).
Applying the same formulas as used in A2704-12 case, we have the distribution of A5547-127 (beans through the ordered aliquots) $1,1,1,1,1,1,1,2,3,4$ and the total A5547-127 content in the 3000 seed lot $\mathrm{N}=16$. Again, this is the exact match with the actual distribution in the seed lot, both for each aliquot and for the total presence of the target seeds.

$n_{\max }$ and $n_{\min }$ calculated for each aliquot with $\mathrm{A}=(-3,50-0.16)$ and $\mathrm{A}=(-3,50+0.16)$, respectively, provide $\sigma_{N}=0.59$ and the final result is:

$$
N=16 \pm 0.59 ; N_{\text {actual }}=16
$$

SeedCalc gives $6 \leq N \leq 26$, Confidence Interval $(C I)=95 \%$ with the optimum at $\mathrm{N}=14$.

\section{Analysis without self-reference calibration}

It can be seen from Figure $3\left(\mathrm{P}_{\text {II }}(\mathrm{N})\right)$ that for both $\mathrm{A} 2407-12$ and A5547-127 the criterion is applicable of having exactly one and 
exactly two target seeds in the aliquots with lowest and second lowest presence of target seeds. In this case all the calculations can be done without self-reference calibration because the slope (A) is derived directly from $\mathrm{C}_{\mathrm{t}}$ values for the aliquots with one and two target seeds. Thus, $A=-\left(C_{t}(1)-C_{t}(2)\right) / \log 2$, where $\mathrm{C}_{\mathrm{t}}(1)$ and $\mathrm{C}_{\mathrm{t}}(2)$ are threshold cycles for the aliquots with 1 and 2 target seeds.

It follows from Figure 5 that there are five aliquots with one target seed and three aliquots with two target seeds in case of A2704-12. For A5547-127 it is 7 and 1, respectively. Thus, we have $\mathrm{A}=-3.44 \pm 0.22$ (average of 15) for A2704-12 and $\mathrm{A}=-3.81 \pm 0.30$ for $\mathrm{A} 5547-127$ (average of 7). The average $C_{t}(1)$ are 27.75 and 25.98 , respectively. The calculations with the formulas used above give $\mathrm{N}=14 \pm 0.51$ (A2704-12) and $\mathrm{N}=15 \pm 0.72$ (A5547-127).

This approach, not employing calibration, though convenient and applicable for the wide range of positive aliquots (9-16), is less accurate because the slope is defined by two points only in the most dilute area of target seeds content. This approach should be implied only in case when there are not less than three $C_{t}(1)$ and $C_{t}(2)$ aliquots each.

\section{GTS 40-3-2}

Results for real-time PCR performance on GTS 40-3-2 are given in Figure 6 and Table 1S.
Correspondence between the actual and sorted order of the aliquots can be found in Table 1S.

As it follows from Figure 6, all twenty aliquots showed positive for GTS 40-3-2, which means that the total number (N) of GTS 403-2 beans in the lot of 3000 seeds can be within 35-3000 range, thus, algorithm c) shall be applied, when the pre-median aliquot and the next differing aliquot above median differ by one target seed. $\# 10\left(C_{t}=26.14 \pm 0.15\right)$ is the pre-median aliquot by definition. It can be seen that aliquots \#\#7-14 show $\mathrm{C}_{\mathrm{t}}$ within the error of the pre-median aliquot, thus, shall be considered having equal target seeds content. Then, the average $\mathrm{C}_{\mathrm{t}}$ for the pre-median aliquot is 26.14. \#15 $\left(C_{t}=25.93 \pm 0.06\right)$ is the first after the median, which has $\mathrm{Ct}$ value that differs from \#10 outside the error. Self-reference calibration through dilutions of aliquot \#20 showed true linearity $\left(\chi^{2}=0.0333\right.$ and $\left.R^{2}=0.9955\right)$ and the slope (A) was $-3.44 \pm 0.13$

Therefore

$$
\mathrm{n}_{1}=\frac{1}{10^{\left(\frac{26.13-25,93}{-3.44}\right)}-1}
$$

and the number of target seeds (GTS 40-3-2 beans) in the premedian aliquot is $\mathrm{n} 1 \approx 6.98=7$.

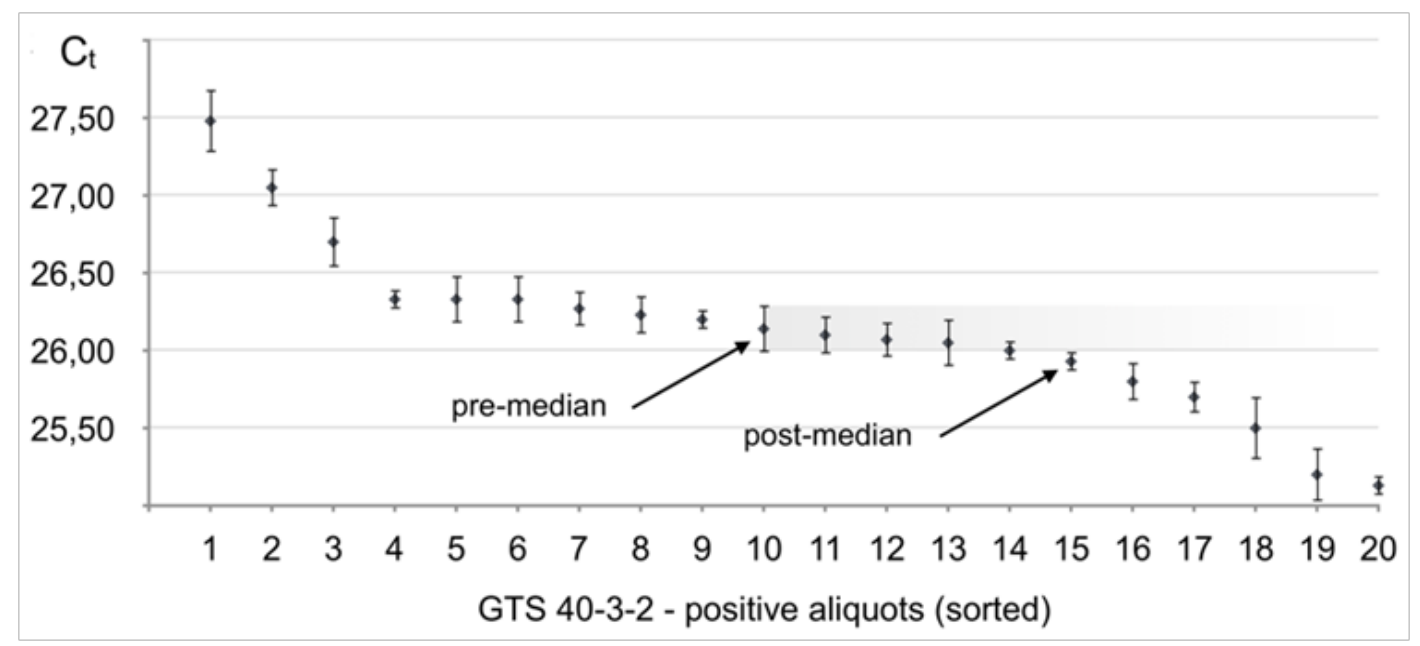

Figure 6 Real-time PCR performance on soybean line GTS 40-3-2 in the seed lot of 3000 seeds distributed in 20 aliquots. Positive aliquots arranged in the order from the lowest target seed content (latest $C_{t}$ ) to the highest (earliest $C_{t}$ ). Error bars give standard deviation values $(\sigma)$ resulting from multiple repeats of testing of each aliquot (Table IS).

Then $n_{i}$ and $\mathrm{N}$ were calculated with the same formulas as for A2704-12 and A5547-127. The distribution of GTS 40-3-2 seeds is given in Figure 7.

The total presence of GTS 40-3-2 in the lot of 3000 seeds was

$$
N=150 \pm 0.54 ; N_{\text {actual }}=150
$$

SeedCalc gives $35 \leq \mathrm{N} \leq \ll$ Error».

Application of the b) criteria of the common algorithm (two aliquots with the lowest target seeds content differ by exactly one target seed) gives close result.

There are some minor deviations between the actual and experimentally estimated distribution of GTS 40-3-2 across the aliquots as it can be seen from Figure 7. Thus, twelve aliquots show the exact match, four aliquots show +1 seed for the actual distribution and four show -1 . The total number of the target seeds is the same in both cases though, which means that the deviations are not system error and can be explained by normal experimental and measuring errors.

The experiment provided practical rationale for setting 150 target seeds as the upper limit of in terms of using real-time PCR. Indeed, $\log [\mathrm{n} / \mathrm{n}+1]$, which determines the difference between two Ct values tends to zero very quickly with increasing $\mathrm{n}$. Thus, the applicability of the approach is determined by the condition

$$
\frac{\mathrm{C}_{\mathrm{t}}(\mathrm{n})-\mathrm{C}_{\mathrm{t}}(\mathrm{n}+1)}{\mathrm{A}} \geq \log \left(\frac{\mathrm{n}}{\mathrm{n}+1}\right)
$$


Our direct calculations checked with Monte Carlo method show that pre-median number varies within the range 2-8 when all 20 aliquots show positive (the number of target seeds in the seed lot of 3000 seeds). In case of 150 target seeds per lot of 3000 seeds probability of occurrence of the particular number of target seeds in the pre-median aliquot reveals a very sharp optimum. It is 0.0098 for 5 target seeds, 0.4461 for $6,0.5115$ for 7 , and 0.0126 for 8 . Probability of having less than 5 and more than 8 target seeds in the pre-median aliquot is vanishingly low. Thus, with the slope (A) value being between -3.0 and -3.5 (most often observed in the experiments) the practical detection limit condition is

$$
C_{t}\left(m^{-}\right)-C_{t}\left(m^{+}\right) \geq 0.17-0.20
$$

where $\mathrm{m}^{-}$and $\mathrm{m}^{+}$is the number of target seeds in the identified premedian and post-median aliquots, respectively. This condition is very close to the experimental and measurement errors. These considerations give us another surprising, though quite expected result-accuracy and reliability of the method grows along with decreasing target seeds content. Indeed, the difference between threshold cycles for aliquots containing $n$ and $(n+1)$ target seeds is much more visible with lower $n$, and it is easier to precisely differentiate between them.

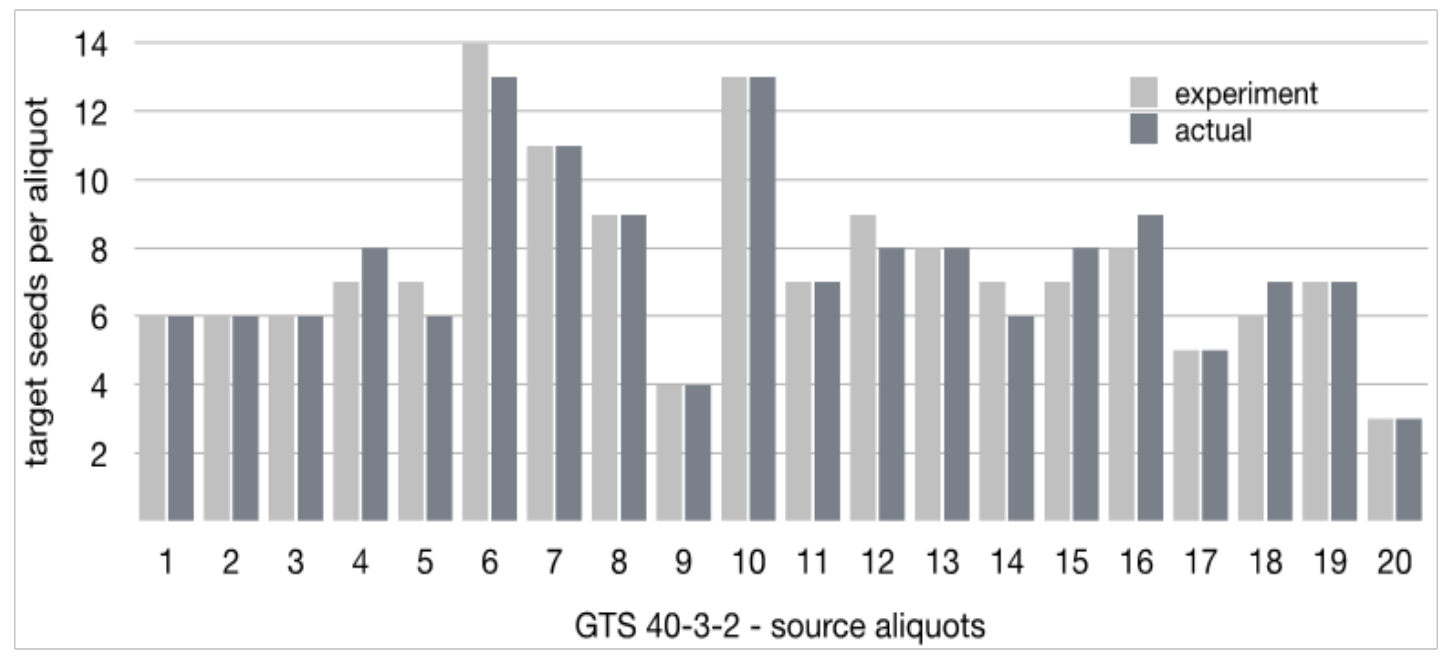

Figure 7 Experimentally estimated vs actual distribution of GTS 40-3-2 seeds in the 20 aliquots in the seed lot of 3000 seeds.

\section{Conclusions}

The proposed approach works correctly (with the confidence $>$ $95 \%$ ) on discreet systems within concentration range $0.033-5 \%$ and can be used without any reference (self-reference calibration) within the wide range of target seed content (0.3-2\%). Since the approach employs seed count mode, it does not depend upon weight difference between bulk and target seeds and eliminates the ambiguity that can arise due to the possible difference in the efficiency of DNA extraction from the target seeds and the certified reference material, as well as the difference in PCR performance. The approach appears to have high potential if combined with digital PCR, when a specific number of copies of the target DNA is assigned to exactly one seed, thus the positive aliquots series becomes plain arithmetic progression, requiring no calibration at all. The lower limit of quantitation can be easily decreased by increasing the size of the initial seed lot and the number of aliquots. Thus, in case of 9000 seeds and 60 aliquots it will be $0.01 \%$ with $\sim 99.97 \%$ confidence, though in many cases it turns impractical due to increasing labor and laboratory costs. Detailed analysis of the connection between detected level of adventitious presence and the costs is given elsewhere. ${ }^{11}$

The proposed approach can be applied not on biotechnology seeds only, but on tracing whatever marker not appearing in the bulk seeds. Thus, we have successfully quantified the occasional presence of millet seeds in live sample of commodity rapeseeds by tracing caat (cytosolic aspartate aminotransferase) gene, with the cross-check of the results with manual seed counting.
We have also successfully experimented with weighing aliquots with the mixture of the balls, that were the same in size but differed in weight, finding the weight of each type of the balls.

And this means that the proposed approach may be applied to whatever physical method of testing, provide there is an established dependence between the observed signal and the concentration of the traced trait.

\section{Conflicts of interest}

There are no conflicts to declare.

\section{Acknowledgements}

The authors express their most sincere gratitude to Mr. Konstantin Kozlovsky ("SunLineStroy" Company) for his fruitful contribution to the theoretical part of the work, and to Mr. Vitaly Smirnov ("Group of Companies SODRUGESTVO”) for providing the material.

\section{References}

1. Mazzara M, Paoletti C, Corbisier P, et al. Kernel Lot Distribution Assessment (KeLDA): A Comparative Study of Protein and DNABased Detection Methods for GMO Testing, Food Analytical Methods. 2013;6(1):210-220.

2. Bogožalec-Košir A, Demšar T, Štebih D, et al. Digital PCR as an effective tool for GMO quantification in complex matrices, Food Chemistry. 2019;294:73-78. 
3. Laffont JL, Remund KM, Wright D, et al. Testing for adventitious presence of transgenic material in conventional seed or grain lots using quantitative laboratory methods: statistical procedures and their implementation, Seed Science Research. 2005;15(3):197-204.

4. Brualdi RA. Introductory Combinatorics. $5^{\text {th }}$ ed, New Jersey: Pearson Prentice Hall; 2010.

5. Joarder AH. Hypergeometric Distribution and Its Application in Statistics. In: Lovric M, editor. International Encyclopedia of Statistical Science, Germany: Springer Berlin Heidelberg; 2011. p. 641-643.

6. Grinstead MC, Snell JL. Introduction to Probability. $2^{\text {nd }}$ Revised ed, USA: American Mathematical Society; 1997.

7. Knuth DE. The Art of Computer Programming: Fundamental algorithms. $3^{\text {rd }}$ ed, USA: Addison-Wesley Professional; 1997.
8. Mazzara M, Delobel C, Grazioli E, et al. Event-Specific Method for the Quantification of Soybean Line A2704-12 Using Real-Time PCR - Validation Report and Protocol - Soybean Seeds Sampling and DNA Extraction. Online Publication. 2007.

9. Delobel C, Bogni A, Mazzara M, et al. Event-specific Method for the Quantification of Soybean Line A5547-127 Using Real-time PCR Validation Report and Protocol. Online Publication. 2009.

10. Mazzara M, Munaro B, Larcher S, et al. Event-specific Method for the Quantification of Soybean Line 40-3-2 Using Real-time PCR - Validation Report and Protocol - Report on the Validation of a DNA Extraction Method for Soybean Seeds, Online Publication. 2007.

11. European Network of GMO Laboratories Working Group "Seed Testing" (WG-ST) Working Group Report, Online Publication. 2015. 